\title{
ESTUDO DO ÍNDICE DE DISFUNÇÃO TEMPOROMANDIBULAR (DTM) EM PACIENTES DA CLÍNICA INFANTIL DA UNIVERSIDADE CIDADE DE SÃO PAULO.
}

\section{STUDY OF THE INDEX OF TEMPOROMANDIBULAR DISORDERS (TMD) IN CHILDREN PATIENTS FROM SÃO PAULO CITY UNIVERSITY}

\author{
Karyna Martins do Valle-Corotti * \\ Paulo Eduardo Guedes Carvalho * \\ Danilo Furquim Siqueira \\ José Roberto Fonseca Junior * \\ Luana de Souza Brito ** \\ Caio Fabre Carinhenas **
}

\begin{abstract}
RESUMO
Introdução: Este estudo tem como objetivo avaliar o índice de Disfunção Temporomandibular em 50 crianças (25 meninos e 25 meninas), da Clínica Infantil da Universidade Cidade de São Paulo. Metodologia: O índice de DTM foi determinado a partir de um questionário anamnésico referente aos sintomas da disfunção. Em seguida os pacientes foram examinados para avaliação dos movimentos mandibulares, da articulação temporomandibular e das características oclusais. Resultados: A amostra, de 3 a 11 anos, apresentou média de idade de 7,9 anos. Foram encontrados $2 \%$ com DTM Moderada; $42 \%$ com DTM Leve e 56\% de DTM Ausente. Não foram encontradas crianças com DTM severa. Conclusão: Os sintomas de DTM podem estar presentes desde a infância e devem ser observados e controlados, a fim de se evitar que os mesmos se agravem com o avançar da idade.
\end{abstract}

DESCRITORES: Síndrome da disfunção da articulação temporomandibular

\section{ABSTRACT}

Introduction: The aim of this study is to evaluate the prevalence of Temporomandibular Disorders (TMD) in 50 childrens. Methods: The patients selected for this study were from São Paulo City University. The TMD index was obtained by an anamnestic questionnaire and the clinical evaluation was composed by the analysis of mandibular movments, temporomandibular joints and occlusal caractheristics. Results: Results showed a prevalence of $42 \%$ of the cases studied with mild TMD, $2 \%$ with moderate TMD and $56 \%$ with absent TMD. Conclusions: The TMD symptoms are present since the childhood and need to be controlled by the orthodontists.

DESCRIPTORS - Temporomandibular joint disfunction syndrome

\footnotetext{
* Professor Associado do Curso de Mestrado em Ortodontia da Universidade Cidade de São Paulo (UNICID).

** Aluno do curso de graduação da Universidade Cidade de São Paulo (UNICID).
}

*** Aluno do curso de mestrado da Universidade Cidade de São Paulo (UNICID). 


\section{N T R O D U Ç Ã O}

As disfunções do sistema mastigatório são, atualmente, alvo de vários estudos presentes na literatura $1,2,3,4,5, \mathrm{O}$ aumento da procura para tratamento de diferentes sintomas relacionados à Disfunção Temporomandibular (DTM) leva a comunidade científica à procura de mais conhecimento quanto aos fatores etiológicos da DTM, a fim de proporcionar ao clínico um diagnóstico mais preciso e um tratamento individualizado nos diferentes casos.

O termo Disfunção Temporomandibular (DTM) compreende alterações clínicas caracterizadas por sinais e sintomas nos músculos da mastigação, na articulação temporomandibular (ATM) ou em ambos (Barros e Rodi ${ }^{6}$ 1995, Grosfild e Czarnecka $^{7}$ 1997, Macnamara et al. ${ }^{8}$ 1995). Essas disfunções apresentam uma etiologia multifatorial o que dificulta a elaboração de uma estratégia de terapia preventiva, pois não é possível determinar até que ponto cada um dos fatores, isoladamente ou em conjunto, indicará o desenvolvimento de uma doença Temporomandibular.

Vários autores encontraram uma alta prevalência de DTM na população, no entanto a grande maioria dos estudos é realizada em jovens e adultos, porém os sinais e sintomas de Disfunção Temporomandibular também aparecem em crianças e adolescentes 9, 2, 10,5,11, . Afirmam ainda que mesmo que esses sintomas sejam geralmente brandos, podem evoluir para severos, provocando Disfunção Temporomandibular com o desenvolver da idade 9, 12, 13, 10,

Não fica claro na literatura se esses sintomas presentes na infância comprometem a vivência da criança e quando devem ser tratados. Com a finalidade de avaliar os sinais e sintomas das DTM em crianças, este trabalho observou a prevalência dos sintomas de DTM em pacientes da Clínica Infantil da Universidade Cidade de São Paulo.

\section{REVISÃO DA LITERATURA}

Observou-se um aumento significativo nas publicações a partir dos anos 90 . Os estudos mostram grande variação na idade da população pesquisada, tendo como consequência uma grande variação nos resultados.

Grosfeld e Czarnecka7 (1977) observaram uma incidência de 56,7\% e $67,6 \%$ de disfunções temporomandibulares nos grupos de crianças com 6 a 8 anos e 13 a 15 , respectivamente. Porém, não encontraram relação entre as maloclusões e o desenvolvimento das DTMs.

Egermark-Eriksson et al. ${ }^{14}$ (1981) estudaram três grupos de pacientes, com idades de 7, 11 e 15 anos, cada grupo. A prevalência dos sintomas encontrados nessa pesquisa foi de $39 \%$ no grupo de crianças com 7 anos, $67 \%$ no grupo com 11 anos e $74 \%$ no grupo com 15 anos. Concluíram que a maloclusão funcional, com interferências oclusais, foi muito mais importante na etiologia das DTMs do que a maloclusão morfológica.

Greene $^{15}$ (1982) concluiu que a correção ortodôntica de más oclusões dentoesqueléticas reduz as chances de desenvolvimento de DTM no futuro. No entanto, comentou que o tratamento ortodôntico não pode ser considerado como uma terapia específica para a DTM.

Bernal e Tsamtsouris ${ }^{16}$ (1986) examinaram clinicamente 149 crianças, entre 3 e 5 anos, e aplicaram um questionário nos pais dessas crianças. Observaram que sintomas subjetivos foram relatados pelos pais em 38\% das crianças, e a maior prevalência foi dor de ouvido e ranger de dentes. No mínimo um sinal de DTM foi encontrado em $36,4 \%$ do total da amostra. A maior prevalência foi de alterações no padrão de movimento dos côndilos, que apareceram em $20 \%$ do total, podendo tal fato ser atribuído a mudanças no contorno da ATM, o que está ocorrendo nessa faixa etária. A prevalência de ruídos articulares foi mais baixa do que em outros estudos, sendo registrada em $5 \%$ dos casos. Contudo, houve um aumento na frequência desse sinal com o aumento da idade. Foi encontrada, também, uma correlação positiva entre idade e desgaste dentário.

Bromberg et al. ${ }^{9}$ (1992) obervaram 100 crianças entre 6 e 15 anos em tratamento ortodôntico. Constataram que, das 100 crianças, 19 apresentaram problemas de ATM e que os problemas mais frequentes foram: estalido na abertura e fechamento
VALLE-COROTTI KM CARVALHO PEg SIQUEIRA DF

FONSECA JUNIOR JR

BRITO LS

CARINHENAS CF

ESTUDO DO ÍNDICE

DE DISFUNÇÃO

TEMPOROMANDIBULAR

(DTM) EM PACIENTES

DA CLÍNICA

INFANTIL DA

UNIVERSIDADE

CIDADE DE SÃO

PAULO.
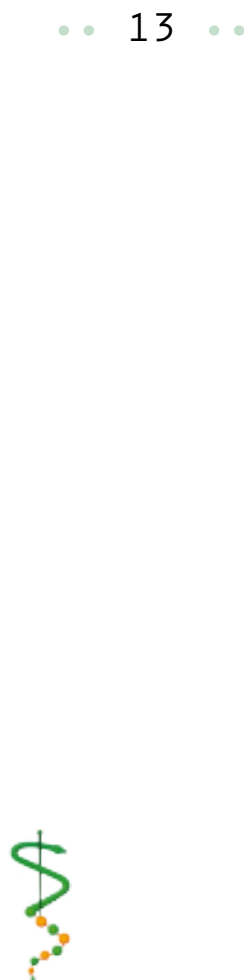

REVISTA DE

ODONTOLOGIA DA

UN I VERS I DADE

CIDADE DE São

PAULO

$2010 ; 22(1): 12-8$ JAN - ABR 
VALLE-COROTTI KM

CARVALHO PEG

SIQUEIRA DF

FONSECA JUNIOR JR :

BRITO LS

CARINHENAS CF

ESTUDO DO INNICE

DE DISFUNÇÃO

TEMPOROMANDIBULAR

(DTM) EM PACIENTES :

DA CLÍNICA

INFANTIL DA

UNIVERSIDADE

CIDADE DE SÃO

PAULO.

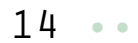

REVISTA DE

ODONTOLOGIA DA

UNI VERS I DADE

CIDADE DE SÃO

PAULO

$2010 ; 22(1): 12-8$,

$J A N-A B R$ da boca, dores de cabeça, dor ou zumbido na região dos ouvidos, desvio da linha média, bruxismo e limitação na abertura da boca.

Deng et al. ${ }^{17}$ (1995), em um estudo com 3105 crianças chinesas entre 3 e 19 anos, acharam uma prevalência de DTM de $17,9 \%$, não sendo encontrada diferença significativa entre os sexos. Os sinais de DTM apareceram em maior número nas crianças com dentição mista e no início da dentição permanente. Os sinais mais comuns encontrados foram ruídos articulares (detctados seguidos em 87,7\%), seguidos de movimentos mandibulares anormais $(23,6 \%)$. Dor foi registrada em apenas 0,6\% da população.

Barone et al. ${ }^{18}$ (1997) analisaram 240 crianças para estimar a prevalência de DTM e a necessidade de tratamento funcional. A amostra foi dividida em dois grupos: os que necessitavam de tratamento ortodôntico e os que não necessitavam de tratamento ortodôntico. Concluíram que a alta prevalência de sinais e sintomas de DTM não foi realmente a razão da disfunção mastigatória e que má oclusão não pareceu causar DTM e/ou aumentar a necessidade de tratamento ortodôntico em crianças jovens.

Widmalm et al. ${ }^{19}$ (1999) examinaram 540 crianças em idade pré-escolar, à procura de alguma associação entre os sintomas das DTMs e os ruídos articulares. A prevalência dos ruídos articulares, encontrada em toda a amostra (fossem eles auscultados pelos profissionais ou relatados pelas próprias crianças), foi de 16,7\%. Houve uma associação significativa entre a crepitação e a dor na região da ATM e do músculo masseter, o que não foi observado quando na presença dos estalidos articulares.

Coutinho et al. ${ }^{20}$ (2003), por meio de uma revisão da literatura, estudaram os efeitos do tratamento ortodôntico sobre a ATM, e se este contribui para o aparecimento da DTM. Não ficou provado cientificamente que o tratamento ortodôntico isoladamente seja a causa da DTM. Como a etiologia mostrou-se multifatorial, foi necessário levar em consideração: o crescimento, a má oclusão morfológica e funcional, os fatores psicológicos, emocionais e estresse para se alcançar um diagnóstico final.

Muhtarogullat et al. ${ }^{13}$ (2004) avaliaram a prevalência de sinais e sintomas de DTM em 40 crianças com dentição decídua e em 40 crianças com dentição mista. A amostra foi avaliada clinicamente e pela aplicação de um questionário. Os resultados demonstraram um aumento nos sinais e nos sintomas da dentição decídua para a dentição mista, mas apenas os ruídos articulares foram estatisticamente diferentes entre os dois grupos.

Santos et al. ${ }^{21}$ (2006) avaliaram a frequência dos sinais e sintomas dos hábitos parafuncionais e das características oclusais de 80 crianças e adolescentes de 5 a 12 anos e concluíram que os sinais e sintomas mais frequentes foram o hábito de onicofagia $(47,5 \%)$, ranger os dentes (35\%), dores de cabeça $(22,5 \%)$ e ruídos na ATM (8,7\%). Diante disso, torna-se aconselhável que a avaliação de sinais e sintomas da disfunção da ATM em crianças seja adotada como rotina durante o exame clínico inicial. Os autores realizaram uma revisão da literatura sobre a relação entre a DTM e o bruxismo em crianças e adolescentes. Observaram que a prevalência da DTM variou grandemente e os sinais e sintomas mais comuns foram: sons articulares, limitação de abertura bucal, dores de ouvido, dores faciais, dores de cabeça. Comentaram que a etiologia da DTM e do bruxismo é controversa, mas multifatorial, incluindo aspectos patofisiológicos, psicológicos e morfológicos. Comentaram ainda que, em crianças, o bruxismo pode ser uma consequência da imaturidade do sistema mastigatório neuromuscular.

Devido à diversidade de dados presentes na literatura e à escassez de dados sobre a população brasileira, optou-se pela avaliação dos sintomas de DTM em uma amostra composta por crianças brasileiras.

\section{Proposição}

Com base na literatura, consultada este trabalho se propõe a avaliar o índice e a prevalência dos sintomas de Disfunção Temporomandibular em crianças pertencentes à Clínica Infantil da UNICID. 


\section{MATERIAL E MÉTODOS}

A amostra foi composta de 50 crianças, pertencentes à Clínica Infantil da Universidade Cidade de São Paulo, com idade entre 3 e 11 anos.

Não participaram da amostra pacientes com síndromes ou discrepâncias esqueléticas severas decorrentes de malformações congênitas.

Foi aplicado um Questionário Anamnésico respondido pelos pacientes da Clínica de Ortodontia e Odontopediatria da UNICID com a colaboração dos pais ou responsáveis. O questionário apresentava questões relacionadas aos sintomas de Disfunção Temporomandibular. O exame clínico foi realizado por apenas um examinador.

\section{Questionário Anamnésico}

1. Você sente dificuldade de abrir a boca?

2. Você sente dificuldade de movimentar sua mandíbula para os lados?

3. Você sente desconforto ou dor muscular quando mastiga?

4. Você sente dores de cabeça com frequência?

5. Você sente dores no pescoço e/ou ombros?

6. Você sente dores de ouvido ou próximo a ele?

7. Você percebe algum ruído na ATM?

8. Você usa apenas um lado de sua boca para mastigar?

9. Você sente dores na face ao acordar?

Foram oferecidas as possibilidades das respostas: "sim", "não" ou "às vezes". Cada "sim" recebeu o valor 2, "às vezes" valor 1 e zero para a resposta "não", com base no Questionário utilizado por ValleCorotti et al. ${ }^{22}$ (2003).

A somatória dos valores obtidos permitiu a classificação da amostra em relação à DTM em:

1. Valores de 00 a 03: não portador de DTM;

2. Valores de 04 a 08: portador de DTM leve;

3. Valores de 09 a 13: portador de
DTM moderada;

4. Valores de 14 a 18: portador de DTM severa.

Durante a anamnese o paciente foi ainda questionado com relação à presença de hábitos como ranger os dentes, apertar os dentes, roer unhas, morder objetos (canetas, lápis), mascar chicletes com frequência, chupar chupeta, chupar dedo, e tomar mamadeira.

\section{Exame Físico}

O exame físico foi realizado por um examinador, para a obtenção dos dados relacionados à avaliação da ATM e da oclusão.

\section{Avaliação da ATM}

A ATM foi avaliada pelos movimentos mandibulares de abertura e fechamento, que eram simétricos, com desvio ou com deflexão. A presença de estalido foi observada por uma suave palpação bilateral na ATM.

\section{Avaliação Dentária e Oclusal}

Todos os pacientes da amostra foram avaliados quanto ao desenvolvimento da dentição e deveriam apresentar dentadura decídua ou mista.

O exame da oclusão constou da avaliação das más oclusões no sentido ânteroposterior (Classe I, II e III); vertical (mordida aberta anterior); transversal (mordida cruzada posterior), overjet, overbite, presença de facetas de desgaste.

\section{RESULTADOS}

A amostra foi realizada em 50 pacientes (25 do sexo feminino e 25 do sexo masculino), entre 3 e 11 anos de idade, com uma média de idade de 7,96 anos. Em relação à má oclusão sagital a amostra apresentou 60\% Classe I; 30\% Classe II; $10 \%$ Classe III. Observou-se ainda que $18 \%$ dos pacientes apresentavam mordida aberta anterior, e 20\% apresentavam mordida cruzada posterior, sendo, destes, $40 \%$ bilateral, e $60 \%$ unilateral.

De acordo com as respostas obtidas no questionário anamnésico, observou-se a seguinte classificação de DTM: $2 \%$ com DTM Moderada; 42\% com DTM Leve e $56 \%$ de DTM Ausente. Não foram encontradas crianças com DTM severa.

Entre os sintomas avaliados no questio-
VALLE-COROTTI KM

CARVALHO PEG

SIQUEIRA DF

FONSECA JUNIOR JR

BRITO LS

CARINHENAS CF

ESTUDO DO ÍNDICE

DE DISFUNÇÃO

TEMPOROMANDIBULAR

(DTM) EM PACIENTES

DA CLÍNICA

INFANTIL DA

UNIVERSIDADE

CIDADE DE SÃO

PAULO.

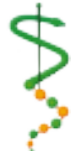

REVISTA DE

ODONTOLOGIA DA

UN I VERS I DADE

CIDADE DE SÃO

PAULO

$2010 ; 22(1) ; 12-8$ JAN - ABR 
VALLE-COROTTI KM

CARVALHO PEG

SIQUEIRA DF

FONSECA JUNIOR JR :

BRITO LS

CARINHENAS CF

ESTUDO DO INNICE

DE DISFUNÇÃO

TEMPOROMANDIBULAR

(DTM) EM PACIENTES

DA CLÍNICA

INFANTIL DA

UNIVERSIDADE

CIDADE DE SÃO

PAULO.

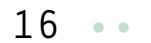

REVISTA DE

ODONTOLOGIA DA

UNI VERSIDADE

CIDADE DE SÃO

PAULO

$2010 ; 22(1): 12-8$,

$J A N-A B R$ nário dos anamnésico, os mais frequentes foram: dos $68 \%$ dos pacientes que mastigavam apenas com um lado da boca, 36\% apresentavam dores de cabeça e 32\% apresentavam dor ou algum desconforto quando mastigava. Os estalidos na articulação temporomandibular ocorreram em $6 \%$ de todo o grupo.

Com relação aos hábitos parafuncionais, $62 \%$ dos pacientes não apresentavam nenhum tipo de hábito; $36 \%$ apresentavam até 3 hábitos parafuncionais e apenas $2 \%$ apresentavam mais que 3 hábitos parafuncionais.

Observando-se os movimentos mandibulares, 94\% tinham uma abertura simétrica; $4 \%$ tinham uma abertura com deflexão; e 2\% uma abertura com desvio.

\section{I SCUSSÃO}

A amostra do presente estudo foi obtida na Clínica Infantil da Universidade Cidade de São Paulo e avaliada por um único examinador. Todos os pacientes foram examinados clinicamente para a avaliação da presença de más oclusões, presente em $60 \%$ da amostra, no entanto não foram comparados os resultados das más oclusões com o índice de DTM, visto que a literatura (Calheiros et al. ${ }^{1}$ 2003, Larson e Ronnerman ${ }^{23}$ 1981, Valle-Corotti et al. ${ }^{22}$ 2003), relata não correlação entre a má oclusão e a DTM.

A prevalência dos sinais e sintomas da DTM foi avaliada em diferentes populações que demonstraram uma variedade de resultados, o que sugere que a DTM parece estar presente em grande parte da população, independente da idade na amostra estudada. Na literatura foram encontrados trabalhos que demonstraram prevalência de sintomas variando de 13\% a $74 \% 16,17,7,24,21$. No entanto esses trabaIhos não classificaram a amostra por índice, provavelmente por terem estudado uma amostra infantil ou adolescente, pois o índice é comumente usado em amostra de indivíduos adultos ou jovens (Keverkari et al. ${ }^{3}$ 1998, Valle-Corotti et al. ${ }^{22} 2003$, Wildmalm et al. ${ }^{19}$, 1999).

Por não existir uma limitação do uso de uma avaliação por índice de DTM em uma amostra infantil, foi utilizado no presente trabalho um questionário anamnési- co que possibilitou definir o grau de DTM. Com a média de idade de 7,96 anos, a amostra apresentou $2 \%$ com DTM Moderada; $42 \%$ com DTM Leve e 56\% de DTM Ausente. Não foram encontradas crianças com DTM severa. Considerando a amostra total, $44 \%$ apresentavam algum grau de DTM, porcentagem esta que se assemelha aos resultados de outros trabalhos, valor próximo ao encontrado por Egermark-Eriksson et al. ${ }^{14}$ (1981) em crianças com 7 anos (39\%) (Egemark e Thinlander ${ }^{24} 1992$, Egermark-Eriksson et al. $\left.{ }^{14} 1981\right)$.

Além do índice de DTM, os sintomas presentes no questionário foram avaliados isoladamente, o que permitiu uma melhor comparação com outros trabalhos. O sintoma mais frequente nessa amostra foi o de mastigar com apenas um dos lados da boca (68\%). Esse resultado não se assemeIha aos de outros trabalhos (Deng e Fel ${ }^{17}$ 1995, Santos et al. ${ }^{21}$ 2006) e provavelmente essa alta frequência ocorreu devido ao fato de, na fase de transição entre a dentadura decídua e mista, a ausência de dentes posteriores dificultar a mastigação em um dos lados da boca.

Em seguida, a maior frequência foi do sintoma dor de cabeça, presente em $36 \%$ da amostra. Outro estudo (Santos et al. ${ }^{21}$ 2006) demonstrou a presença de dor de cabeça em 22,5\% e, embora as frequências não sejam semelhantes entre os estudos, observa-se que esse é um sintoma comum também em crianças e que merece atenção desde a infância.

Os ruídos articulares foram encontrados em apenas 6\% da amostra deste estudo, valor próximo ao encontrado por Santos et al. ${ }^{21}$ (2006), (8,7\%), mas inferior ao demonstrado por Deng et al. ${ }^{17}$ (1995), $(87,7 \%)$; entretanto sua amostra apresentou idade bem superior à deste trabalho. Esse sintoma, quando relatado pelo paciente, como os demais, deve ser analisado pelo profissional, pois pode indicar uma alteração estrutural na Articulação Temporomandibular da criança, que pode se agravar com o avançar da idade.

A presença de hábitos foi observada em $62 \%$ da amostra e parece que nessa faixa etária os hábitos não são incomuns, como foi também demonstrado em outros estu-

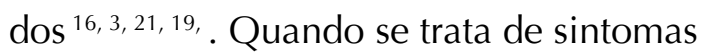


de DTM, os hábitos merecem atenção, pois a literatura relata correlação significante entre bruxismo, onicofagia, sucção digital e a maioria dos sinais e sintomas de DTM em crianças.

A alteração nos movimentos mandibulares indica alteração no relacionamento côndilo-disco da ATM $^{22}$ ou na função dos músculos mastigatórios. Esse sinal foi encontrado em $6 \%$ da amostra, bem inferior ao encontrado por Bernal e Tsamtsouris ${ }^{16}$ (20\%), Deng et al. ${ }^{26}$ (1995) (23,6\%). Porém Bernal e Tsamtsouris ${ }^{16}$ (1986) sugeriram que nessa faixa etária podem ocorrer alterações no contorno da ATM.

Os resultados encontrados nesta pesquisa confirmam a presença de sintomas de Disfunção Temporomandibular em crianças. A boa notícia consiste em que poucos casos apresentam necessidade de tratamento, pois não foram encontradas crianças com índice severo de DTM com DTM moderada, e apenas $2 \%$ da amostra. Esse resultado indica que na maioria dos casos existe a necessidade apenas de esclarecimento aos pais e de acompanhamento dos sintomas, o que pode evitar que estes se agravem com o avançar da idade.

\section{CONCLUSÃO}

$\mathrm{Na}$ amostra avaliada foram encontradas 2 \% com DTM Moderada; $42 \%$ com DTM Leve e $56 \%$ de DTM Ausente. Não foram encontradas crianças com DTM severa. Dentre os sintomas observados na amostra, os mais frequentes foram $68 \%$ dos pacientes que mastigavam apenas com um lado da boca, 36\% apresentavam dores de cabeça e $32 \%$ apresentavam dor ou algum desconforto quando mastigavam. Os estalidos na Articulação temporomandibular ocorreram em $6 \%$ de todo o grupo.

Os sintomas de DTM podem estar presentes desde a infância e devem ser observados e controlados, a fim de se evitar que se agravem com o avançar da idade.

\section{REFERÊNCIAS}

1. Calheiros AA, Sekito PSC, Soares TM, Souza H M MR. Inter-relação entre ortodontia e disfunção têmporo-mandibulares. Rev Bras Odontol 2003 jul-ago; 60(4): 270-3.

2. Hara EB, Camargo FG, Attizzani A. Sinais e sintomas das disfunções temporomandibulares em crianças. Odontol USF. 1998 jan-dez; (16): 91-102.

3. Kirveskari P, Jamsa T, Alanen P. Occlusal adjustment and the incidence of demand for temporomandibular disorder treatment. J Prosthet Dent 1998 Apr; 79 (4): 433-8.

4. Ricketts RM. Clinical implications of the temporomandibular joint. Am J Orthod. 1966 Jun; 52(6): 416-39.

5. Rocha JC, et al. Estudo radiográfico da assimetria da fossa mandibular em crânios secos de crianças. Rev Odontol Unesp 2000 jan-dez; 29(1/2): 205-19.

6. Barros JJ, Rode SM. Tratamento das disfunções craniomandibulares - ATM. São Paulo: Santos, 1995; p.101-116.

7. Grosfeld O, Czarnecka B. Musculo-articular disorders of the stomatognathic system in school children examined according to clinical criteria. J Oral Rehabil. 1997 Apr; 4 (2): 193-200.

8. McNamara JA, Seligman DA, Okeson JP. Occlusion, Orthodontic treatment, and temporomandibular disorders: a review. J Orofac Pain.1995; 9 (1): 73-90.

9. Bromberg T K, Sagretti OMA, Guedes Pinto, A C. Sinais e Sintomas de disfunção crânio-mandibular em crianças e adolescentes em tratamento ortodôntico. Rev Odontopediatr 1992 jul-set; 1(3): 173-81.

10. Pascoal MI N, Rapoport A, Chagas JFS. Prevalência dos sintomas otológicos na desordem temporomandibular: estudo de 126 casos. Rev Bras Otorrinolaringol. 2001 set-out; 67(5): 627-33. 
VALLE-COROTTI KM

CARVALHO PEG

SIQUEIRA DF

FONSECA JUNIOR JR

BRITO LS

CARINHENAS CF

ESTUDO DO ÍNDICE

DE DISFUNÇÃO

TEMPOROMANDIBULAR

(DTM) EM PACIENTES

DA CLÍNICA

INFANTIL DA

UNIVERSIDADE

CIDADE DE SÃO

PAULO.

18

11. Rubio G, Pena L, Mayorga C. Prevalence of Temporomandibular Dysfunction and its association with malocclusion in children and adolescents: an epidemiologic study related to specified stages of dental development. Angle Orthod 2001 Apr; 72 (2): 146-54.

12. Motegi E. et al. An Orthodontic study of temporomandibular joint disorders. Part. 1: Epidemiological research In Japanese 6-18 years old. Angle Orthod 1992; 62 (4): 249-56.

13. Muhtarogullari M, Demirel F, Saygili G. Temporomandibular disorders in Turkish children with mixed and primary dentition: prevalence of sings and symptoms. Turk Pediatr 2004 apr-jun; 46(2): 159-63.

14. Egermark-Eriksson I, Ingervall B, Carlsson GE. Prevalence of mandibular dysfunction and orofacial parafunction in 7-11- and 15- years old Swedish children. Eur J Orthod.1981; 3(3):163-72.

15. Greene CS. Etiologia das disfunções têmporo-mandibulares: atualidades em Ortodontia. Editorial Premier; 1997: 207-212.

16. Bernal M, Tsamtsouris A. Signs and symptoms of temporomandibular joint dysfunction in 3 to 5 years old children. J Pedod. 1986; 10 (2): 127-40.

17. Deng Y, Fu MK.. Occlusal contact changes before and after orthodontic treatment of a group of child \& adolescent patients with TMJ disturbance. Aust Orthod J 1995 mar; 13(4): 231-7.

18. Barone A, Sbordone L, Ramaglia L. Craniomandibular disorders and orthodontic need in children. J Oral Rehabil. 1997 Jan; 24(1): 2-7.

19. Widmalm SE, Gunn SM, Chistiansen RL. Crepitation and clicking as signs os TMD in preschool children. Cranio 1999 Jan; 7(1):58-63.

20. Coutinho MEP, et al. Os efeitos do tratamento ortodôntico sobre articulação têmporo-mandibular. RGO . (Porto Alegre) 2003 out; 51(4): 335-342.

21. Santos ECA, Bertoz FA, Pignatta LMB, Arantes FM. Avaliação clínica de sinais e sintomas da disfunção temporomandibular em crianças. Rev Dent Press Ortodon Facial. 2006; 11 (2): 29-34.

22. Valle-Corotti K M et al. A oclusão e sua relação. Rev Dent Press Ortodon Ortop Facial 2003 nov-dez; 8(6): 79-87.

23. Larsson E, Ronnerman A. Mandibular dysfunction symptoms in orthodontically treated patients tem years after the completion treatment. Eur J Orthod 1981; 3 (2): 89-94.

24. Moraes CS, Köhler GI, Petrelli E. Disfunções Temporomandibulares na população pediátrica e hebeátrica: uma revisão de literatura. J Bras Ortodon Ortop Facial. 2001 set-out; 6(35): 395-401.

25. Egermark I, Thilander B. Craniomandibular disorders with special reference to orthodontic treatment: an evaluation from childhood to adulthood. Am J Orthod Dentofacial Orthop. 1992 Jan; 101 (1): 28-34.

26. Deng YM, Fu MK, Hagg U. Prevalence of temporomandibular joint dysfunction in Chinese children and adolescents. A cross-sectional epidemiological Study. Eur J Orthod.1995 Aug; 17 (4): 305-9.

Recebido em: 31/08/2009

Aceito em: 11/01/2010 